\title{
Power and Robustness in Product-Moment Correlation
}

\author{
Robert I. Fows? \\ Univers:
}

\begin{abstract}
The power of statistical tests based on four popular product-moment correlation coefficients was examined When relatively small samples $(10 \leqslant N \leqslant 100)$ are drawn from bivariate populations of several different distributional shapes. Analytical procedures for determining theoretical power under conditions of bivariate nomalinîy are presented for the Pearson $\left(r_{p}\right)$, Spearman $\left(r_{s}\right)$, point-biserial $\left(r_{\mathrm{pb}}\right)$, and phi $\left(r_{\mathrm{fp}}\right)$ coefficients. A monte carlo siudy supported previous conclusions that
\end{abstract}

\begin{abstract}
$t$ as a test of $\mathrm{H}_{0}: \mathrm{p}=0$, with $r_{\mathrm{p}}$ estimating $p$, is robust over a wide range of non-normality; however, frequent use of $r_{s}$ leads to greater power under identical distributional assumption violations. The proportion of power due to Type III errors was also specified both analytically and empirically, and revealed the relative invilnerability of most statistical tests to directional misinterpretation.
\end{abstract}

Psychometric models are often built on conrelation statistics which require that certain distributional assumptions be met. The empirical robustness of the Pearson $r$ has been investigated extensively for the situation in which the population correlation $p=0$ (Edgell \& Noon, 1984; Havlicek \& Peterson, 1977). The consensus apparendy is that when $X$ and $Y$ are independent variables, the sample correlation coefficient is relatively insensifive to violations of the normality assumption when tested by

$\hat{s}=r_{\mathrm{p}}\left[(N-2) /\left(1-r_{\mathrm{P}}^{2}\right)\right]^{1 / 2}$,

where $N$ is the number of paired $X, Y$ observations in the sample and $r_{\mathrm{P}}$ is the sample Pearson productmoment correlation coefficient. The robustmess of $\tilde{r}_{\mathrm{P}}$ when $\rho \neq 0$ has been examined both analytically (Kraemer, 1980) and empirically (see Kowalski, 1972, for a review), with less agreement as to the effects of mon-normality.

The literature on the robustness of product-moment correlation (PMIC) statistics other than $r_{p}$ is not extensive and tends to be highly technical. Or more interest to asers of statistical tests is a theoretical tradeoff assumed between Type I and Type II errors when distributional assumptions for the theoretically more powerfull test are not met. That is, will the cost in power of ranking or dichotomizing a continuous variable be offset by the additional protection against erroneous rejections of the null hypothesis? Often, the original data are in a form which limits the choice of a PMC statistic, such as when $X$ or $Y$ are inherent dichotomies (e.g., malle or female) or created dichotomies (e.g., normal vs. psychotic).

APPLIED PSYCHOLOGRCAL MEASUREMENT

Vol. 11, No. 4, December 1987, pp. 419-428

(C) Copyright 1987 Applied Psychological Measurement Inc.

0146-6216/871040419-10\$1.75 
Four widely used PMC measures-the Pearson coefficient $\left(r_{p}\right)$, the Spearman tank order coeficient $\left(r_{s}\right)$, the point-biserial coefficient $\left(r_{\mathrm{ph}}\right)$, and the phi-coefficient or four-fold point coefficient $\left(r_{\mathrm{fp}}\right)$-were examined in this study. Other PMCs have been proposed, such as the point-polyserial coefficient (Olsson, Drasgow, \& Dorans, 1982), but are less familiar to researchers and would have increased the scope of the study without contributing much additional information. 值 should also be pointed ont that biserial (polyserial) correlation and tetrachoric (polychoric) correlation are not PMCs; rather, they involve attempts to recapture the estimate of $p$ which is lost (or unavailable) when either $X$ or $\bar{Y}$ is dichotomized (polychotomized) or both $X$ and $\mathbb{Y}$ are dichotomized (polychotomized). For a complete and illuminating discussion of product-moment correlation and various formulas for defining and computing the coefficients, the reader is referred to Nunnally (1978).

When two variables, $X$ and $Y$, are both continuous, or more properly, graduated (Cohen, 1983), then the researcher often has a choice as to which PNC to use. This choice may be based upon considerations such as (1) whether or not certain distributional assumptions about the parent $X, Y$ bivariate population have been met; (2) computational convenience; (3) compatibility of the observed statistic with other statistics in a multivariate model; or (4) whether developing a precise confidence interval for $\beta$ is of greater interest than rejecting $\mathbb{H}_{0}: \rho=0$. Some theorists would argue that if the original data are graduated, any transformation to ranks or categories would be tamtamount to discarding information and is rarely justified (Cohen, 1983; Nunnally, 1978). Perhaps if researchers knew more about potential risk-reward ratios, they could make a more reasoned choice among PMiCs.

The purpose of this study was to examine two types of risk when graduated variables are polychotomized: (1) the loss of power, and (2) an increased probability that a rejected null hypothesis is a Type III error. It will be assumed that both $X$ and $Y$ are originally measured as continuous variables and a choice is available as to which of the four PMC statistics may be applied to the data from a sample. Interest is focused on the effects of distributional violations on the power of these four PMC statistics when applied to the same sample data and evaluated against appropriately chosen critical values of $r$ based upon statistical sampling distributions known to be exact.

\section{Determining Re Relative Tower}

The power efficiency of a statistical test may be evaluated in several ways (Bradley, 1968). A commonly used method of comparing the efficiency of two statistical tests is interms of the ratio of their respective sample sizes to obtain equal power. For example, if $r_{\mathrm{P}}$ required a sample size of 912 to detect an effect size in the population for which $r_{s}$ required a sample size of 1,000 to detect, then the relative efficiency of $r_{\mathrm{S}}$ would be .912 . This value $\left(9 / \mathrm{Fr}^{2}\right)$ is approximately the theoretical asymptotic relative efficiency of the Speaman $r_{S}$ compared to the Pearson $r_{P}$ (Siegel, 1956), although this may only be true when $p=0$ (Wainer \& Thissen, 1976). However, because the theoretical assumptions are never realized in practice, such an index is not very meaningful to the practical researcher trying to decide whether to use one or the other of these tests. That is, most researchers are interested in detecting moderate effect sizes with finite sample sizes rather than effect sizes approaching 0 with infinise sample sizes.

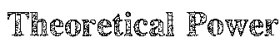

Power values for $r_{P}$ are given for both one- and two-tailed tests in the extensive power analysis tables of Cohen (1977). However, these power values for two-tailed tests include the Type III error rates. A Type III error, or gamma error (Kaiser, 1960), is said to occur when a researcher correctly rejects the null hypothesis and subsequently draws a false conclusion either about the ranking of the parameters 
listed in the embraced altermative hypothesis or about the relationship of a parameter to zero. In the case of PMC coefficients, the usual null hypothesis being tested is $H_{0}: p=0$. If the true situation is $H_{1}: p>0$ and the researcher concludes that $p<0$ because the observed $r$ in the sample is less than the critical value of $r$ in the left tail of its probability distribution, then a Type III error has been made.

PMCS are inherently indices of both the magnitude and the direction of the relationship between $X$ and $Y$ in the population, and are therefore vulnerable to directional misinterpretation upon inspection. The term real power will be used to indicate that the observed statistic is significant and has the same directional sign as the parameter. When an observed statistic is significant but opposite in sign from the parameter, this will be designated Type III power. Total power is the sum of real power and Type III power.

To obtain separate theoretical estimates of real power and Type III error rates, the following procedure was used. The critical values of $r$ for particular combinations of $N$ and $\rho$, as given by Cohen (1977), were converted to $Z^{\prime}$ by means of Fisher's transformation, $\mathbb{Z}_{c}^{\prime}=\operatorname{arctanh} r_{c}$. Values of $p$ were transformed as

$Z_{\rho}^{\prime}=\operatorname{arctanh} \rho+\frac{\rho}{2(N-1)}\left(1+\frac{5+\rho^{2}}{4 N-4}\right)$.

Real power and Type III error rates were then determined by calculating approximate standard normal deviates (Gayen, 1951) as

$z_{\beta+\gamma}=\left(\mathbb{Z}_{c}^{\prime}-\mathbb{Z}_{\rho}^{\prime}\right) / \hat{\sigma}_{z}$

and

$z_{\gamma}=\left(-\mathbb{Z}_{c}^{\prime}-\mathbb{Z}_{\rho}^{\prime}\right) / \hat{O}_{z}$,

where

$$
\hat{\mathbb{v}}_{z}^{2}=\frac{\left(1+\frac{4-\rho^{2}}{2 N-2}\right)+\left(\frac{22-6 \rho^{2}-3 \beta^{4}}{6 N^{2}-12 N+6}\right)}{N-1} .
$$

For small values of $N$, this procedure gave closer approximations to exact values in the tails of the distributions of $r$ (David, 1954) than did the approximation described by Cohen (1977, p. 458). Positive values of $r_{c}$ yielded the $z$ at approximately the $\beta+\gamma$ percentile of the standard normal curve (probability of making a Type II or a Type IJ error when $p \neq 0$ ), whereas negative $r_{c}$ values yielded the approximate z for gamma rates (Type III errors). For example, with $N=10, \alpha=.05$ two-tailed, and $p=.1, r_{c}=$ $.632, \mathbb{Z}_{c}^{\prime}=.745, Z_{\rho}^{\prime}=.107, z_{1-\beta}=1.70$, and $z_{\gamma}=-2.27$. Thus, real power is approximately .045 and Type III power about .012.

The power of $r_{S}$ relative to $r_{\mathrm{P}}$ when all distributional assumptions of the latter are met can be determined analyrically with modification of a procedure described by Drasgow and Dorans (1982) for polyserial $r$. Because the calculation of $r_{\mathrm{s}}$ involves a conversion of two normally distributed graduated variables, $X$ and $Y$, to ranks or consecutive integers, this is essentially the same as polychotomizing the graduated variables by imposing a set of category boundaries $b_{j}$ on a continuous variable, such that

$X^{\prime}=1$ if $Y<b_{1}$,

$X^{i}=j \quad$ if $\quad b_{j-1} \leqslant X \leqslant b_{j} \quad(j=2, \ldots, g-1)$,

and

$X^{\prime}=g$ if $X>b_{g-1}$. 
At this point, the "new" correlation between $Y$ and $X$ is $q p$, where

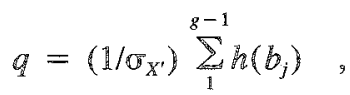

$\sigma_{X^{\prime}}$ is the standard deviation of the $X^{\prime}$ ranks (approximately $3.464 N$ ), and $h$ is the ordinate of the unit normal curve at $b_{j}$. If the polychotomizing procedure is repeated for the $Y$ variable, and assuming both $Y$ and $Y$ were originally normally distributed, the correlation between the integers or ranks in the population will be simply $q^{2} p$. Theoretical power for $r_{S}$ was determined by substinuting $q^{2} p$ for $\rho$ in Equation 2 and substituting critical values of $p_{\mathrm{s}}$ for those of the transformed value of $r_{c}\left(\mathbb{Z}_{c}^{\prime}\right)$ in Equations 3 and 4 . The critical values of $r_{\mathrm{s}}$ for $N=10$ and $N=20$ were derived from Bradley $\left(1968\right.$, p. 314). For $N>20, r_{c}$ is approximately the same for $r_{\mathrm{S}}$ as for $r_{\mathrm{P}}$.

The procedure for determining theoretical power for $r_{\mathrm{pb}}$ and $r_{\mathrm{fp}}$ is computationally more straightorward and is described by Cohen (1983). Dichotomization of one variable (as in $r_{p b}$ ) or of both variables (as in $\left.r_{\mathrm{fp}}\right)$ is the simplest form of the polychotomizing procedure already presented. In this case,

$q_{i}=h_{i} /\left[p_{i}\left(1-p_{i}\right)\right]^{1 / 2}$,

where $p_{i}$ is the proportion of cases in either division of a dichotomized sample, and $h_{i}$ is the normal curve ordinate at the point of dichotomization. For $r_{\mathrm{fp}}$, both variables are dichotomized, not necessarily at the same points on both $X$ and $Y$; hence there are two $q$ values, each based on the respective point at which the particular variable is cut. Thus, power is determined theoretically for $r_{\mathrm{pb}}$ by substituting $q_{i} p$ for $p$ in Equation 2 and applying Equations 3 and 4 . Similarly, the power for $r_{\mathrm{fp}}$ is estimated by substitutuing $q_{1} q_{2} p$ for $\rho$ in Equation 2 and applying Equations 3 and 4 . The critical values for significance of $r_{\mathrm{pb}}$ are the same as those for $r_{p}$, namely $r_{c}$.

It is usually recommended that $r_{\mathrm{ip}}$ be tested for significance as $\chi^{2}=N r_{\mathrm{fp}}^{2}$ with 1 degree of freedom (Ferguson, 1981; Hays, 1981; Nunnally, 1978). Therefore, critical values for $r_{\mathrm{fp}}$ in the present study were derived from the chi-square $(d f=1)$ distribution rather than using the critical values for $r$ based on the $t$ distribution.

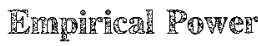

A computer simulation was conducted to determine the effects on PMC power of violating the bivariate normality assumption. The power of the four PMC statistics was assessed at three values of the population correlation coefficient ( $p=.1, .3$, and .5$)$, with four sample sizes $(N=10,20,50$, and 100$)$. These values were chosen because they represent the set of characteristics where power differentials among the statistics would be greatest, while still covering the entire range of theoretical power of the most efficient statistic. That is, the theoretical absolute real power of $r_{\mathrm{p}}$ when all distributional assumptions are met ranged from a low of .008 for $\rho=.1, N=10, \alpha=.01$ to a high of $>.999$ at $\rho=.5, N=100, \alpha=$ .05 . The weakest statistic, $r_{\mathrm{fp}}$, ranged in real power from .005 to .948 . The point-biserial and phi coefficients were each evaluated at five points of cut, with $.1, .3, .5, .7$, or .9 of the sample falling below the point of $Y$ dichotomization. For $r_{\mathrm{fp}}, X$ was dichotomized at the theoretical population median. No ties were permitted in determining the ranks of $X$ and $Y$ for calculating $p_{s}$.

With infinite sample sizes, relative efficiency is independent of the alpha level. However, with the moderate sample sizes typical of many applied measurement studies, the level of significance plays more of an important role in the power comparison of PMC statistics. Accordingly, the relative power of the four PMC statistics was evaluated at the two-tailed alpha levels of .10, .05, and .01. This also permitted the assessment of relative rates of Type III errors for each PMC statistic. This is a facet of power analysis which is usually overlooked when robustness is examined empirically. 
The values of $\rho=.1, .3$, and .5 were chosen to represent a range of correlation coefficients to be found in most behavioral research, and correspond to Cohen's (1977) small, medium, and large effects, respectively. Population correlations greater than .5 are not only rare in practical psychology, but they present more problems than smaller effects in making power comparisons. That is, with large effects, even a weak statistical test will reject the null hypothesis and there is little other than convenience on which to base a choice.

\section{Creation of $\mathrm{Z}_{\mathrm{E}}$ mpirican Population Shapes}

To test the effects of distributional assumptions, various population shapes were created using a series of unit uniform pseudorandom numbers, $u_{i}$. Listed below are the distributions examined and the methods used for simulating their variates.

1. Normal or Gaussian $(G), G_{i}=\left(-2 \log _{e} u_{1}\right)^{1 / 2} \cos 2 \pi u_{2}$, which is the Box and Muller (1958) technique;

2. Uniform $(U), U_{i}=u_{i}-.5$;

3. Exponential $(\mathbb{E}), \mathbb{E}_{i}=-\log _{e} u_{i}+.693142$ and $\mathbb{E}_{i}^{-}=-\mathbb{E}_{i}$;

4. Lognormal $(E), L_{i}=\exp \left[\left(G_{i}-1\right) / .2161197\right]$;

5. LaPlace or double exponential $(D), D_{i}=\mathbb{E}_{i}(-1)^{i}$;

6. Cauchy $(C), C_{i}=G_{i} / G_{i+1}$;

7. Truncated normal or half normal $(H), H_{i}=\left|G_{i}\right|$;

8. The mixed normal $(M)$, simulated by sampling from $N(0, \mathbb{1})$ with probability .9 , from $\mathbb{N}(0,9)$ with probability .095 , and from $\mathbb{N}(0,100)$ with probability .005 . This distribution is also known as a contaminated normal and, except for all the means being 0 so that a theoretical median of 0 could be specified, the mixed normal was similar to one of Bradley's (1977) bizarre but real data distributions.

The $X_{i}$ member of an $X, Y$ pair was then set equal to one of the foregoing $\mathbb{V}_{i j}$ variates, where $j$ is one of the eight shapes. Then the general relationship

$\Psi_{i j}=\rho X_{i j}+\left(1-p^{2}\right)^{1 / 2} V_{i j}$

was used to produce the second member of an $X, Y$ pair, where $V_{i j}$ was from a distribution with the same characteristics as $X_{i j}$ except when skewness was opposite in direction. This regression-produced population correlation technique is similar to that of Duncan and Layard (1973).

For all distributional shapes, the theoretical population median of $X$ was 0 , but the marginal distribution of $Y$ was not always the same as that of $X$ because of the regression technique used. Although this did not result in identical marginal distributions for $X$ and $Y$ when $\rho \not 00$, it did result in the expected value of the empirical linear correlation in the population being reasonably close to the nominal value of p for most of the distributional shapes generated. Because the focus of this study was on relative power of the four PMC statistics when evaluating the same basic data, sample by sample, agreement between the population correlation and the finite expected value was of no great concern.

Table 1 shows the skewness-moment ratio (SMR), kurtosis (KTR), and proportion of $X$ variates above the theoretical median of zero $(X>0)$ for the finite distributions of $10,000 X, Y$ pairs evaluated. For each combination of population correlation and distribution shape, 5,000 cases were generated for $N=10$, 20 , and 50, and 3,000 cases were run for $N=100$. Larger $N$ s were not included because of the excessive computer time involved in the rank ordering process.

\section{Resulits}

The relative real power outcomes for $r_{\mathrm{s}}, r_{\mathrm{pb}}$, and $r_{\mathrm{fp}}$ were consolidated over the three population correlation values and are given in Table 2 for the .05 and .01 levels of significance. For brevity, the .3 
Table 1

Empirical Characteristics

of Populations Studied

\begin{tabular}{|c|c|c|c|c|}
\hline \multicolumn{5}{|c|}{ Distribution } \\
\hline $\begin{array}{l}\text { Shape and } \\
\text { Attribute }\end{array}$ & $\begin{array}{c}\mathrm{X} \\
\text { Variable }\end{array}$ & $\rho=.1$ & $\rho=.3$ & $3 p=.5$ \\
\hline \multicolumn{5}{|l|}{$G, G$} \\
\hline SMR & .004 & .006 & .006 & .001 \\
\hline KTR & 2.84 & 2.83 & 2.82 & 2.83 \\
\hline$X>0$ & .498 & & & \\
\hline \multicolumn{5}{|l|}{$\mathrm{U}, \mathrm{U}$} \\
\hline SMR & -.000 & -.003 & -.007 & -.009 \\
\hline KTR & 1.80 & 1.83 & 2.00 & 2.26 \\
\hline$x>0$ & .507 & & & \\
\hline \multicolumn{5}{|l|}{$\mathrm{E}, \mathrm{E}$} \\
\hline SMR & 1.96 & 1.93 & 1.75 & 1.51 \\
\hline $\mathrm{KTR}$ & 8.32 & 8.20 & 7.38 & 6.27 \\
\hline$x>0$ & .499 & & & \\
\hline \multicolumn{5}{|l|}{$L, \quad L$} \\
\hline SMR & 7.14 & 7.06 & 6.36 & 5.33 \\
\hline KTR & 120.54 & 118.60 & 101.51 & 73.66 \\
\hline$x>0$ & .507 & & & \\
\hline \multicolumn{5}{|l|}{$D, \mathbb{D}$} \\
\hline SMR & -.11 & .13 & -.13 & .10 \\
\hline $\mathrm{KTR}$ & 5.06 & 6.04 & 5.68 & 5.10 \\
\hline$x>0$ & 5.05 & & & \\
\hline \multicolumn{5}{|l|}{$C, C$} \\
\hline SMR & s: & $x^{2}$ & * & $*$ \\
\hline $\mathrm{KTR}$ & 3633 & 6208 & 5496 & 5539 \\
\hline$x>0$ & .501 & & & \\
\hline \multicolumn{5}{|l|}{$\mathrm{H}, \mathrm{H}$} \\
\hline SMR & .96 & .95 & .87 & .76 \\
\hline $\mathrm{KTR}$ & 3.65 & 3.66 & 3.61 & 3.50 \\
\hline$x>0$ & .498 & & & \\
\hline \multicolumn{5}{|l|}{$\mathrm{M}, \mathrm{M}$} \\
\hline SMR & .12 & .14 & .15 & .17 \\
\hline $\mathrm{KTR}$ & 20.13 & 19.78 & 17.45 & 14.10 \\
\hline$x>0$ & .498 & & & \\
\hline \multicolumn{5}{|l|}{$\mathrm{E}^{+}, \mathrm{E}^{-}$} \\
\hline SMR & 2.01 & -1.98 & -1.70 & -1.04 \\
\hline $\mathrm{KTR}$ & 8.92 & 8.81 & 7.95 & 6.73 \\
\hline$X>0$ & .500 & & & \\
\hline
\end{tabular}

HEpirical skewness-moment ratios for the Cauchy distribution are not meaning Iul, but the theoretical value is zero.

and .7 points of cut for $r_{\mathrm{po}}$ were omitted and the .1 and .9 points of cut were combined and designated $r_{\mathrm{pb} .1}$. Only the .5 point of cut is shown for $r_{\mathrm{fp}}$, which had extremely low power otherwise. The number of rejections of $\mathrm{H}_{0}$ for $r_{\mathrm{s}}, r_{\mathrm{pb}}$, and $r_{\mathrm{pp}}$ when the sign of the observed statistic was positive was summed over the three population values $(p=.1, .3$, and .5) separately for each PMC statistic. Then these totals were each divided by the number of rejections of $\mathrm{H}_{0}$ by $r_{\mathrm{P}}$ with positive sign summed over the three population 
Table 2

Real Power as a Percent of Pearson $x$ Power for $N=10,20,50$, and 100

\begin{tabular}{|c|c|c|c|c|c|c|c|c|c|c|c|c|c|c|c|c|}
\hline \multirow{2}{*}{$\begin{array}{c}\text { Shape } \\
\text { and } \\
\text { a }\end{array}$} & \multicolumn{4}{|c|}{$r_{S}$} & \multicolumn{4}{|c|}{$r_{\mathrm{pb} .5}$} & \multicolumn{4}{|c|}{${ }^{r} p b .1$} & \multicolumn{4}{|c|}{$\operatorname{I}_{\mathfrak{f p . 5}}$} \\
\hline & 10 & 20 & 50 & 100 & $\overline{10}$ & 20 & 50 & 100 & 10 & 20 & 50 & 100 & 10 & 20 & 50 & 100 \\
\hline \multicolumn{17}{|l|}{$T$} \\
\hline .05 & 80 & 91 & 95 & 97 & 75 & 73 & 77 & 83 & 56 & 50 & 52 & 61 & 28 & 32 & 40 & 53 \\
\hline .01 & 54 & 78 & 92 & 94 & 68 & 64 & 66 & 75 & 47 & 40 & 37 & 45 & 2 & 15 & 25 & 39 \\
\hline \multicolumn{17}{|l|}{$\mathbb{N}, \mathbb{N}$} \\
\hline .05 & 85 & 89 & 94 & 96 & 72 & 71 & 77 & 84 & 52 & 48 & 50 & 61 & 31 & 46 & 47 & 50 \\
\hline .01 & 71 & 81 & 92 & 95 & 56 & 56 & 67 & 73 & 40 & 35 & 34 & 44 & 12 & 19 & 37 & 39 \\
\hline \multicolumn{17}{|l|}{$\mathrm{U}, \mathrm{U}$} \\
\hline .05 & 89 & 89 & 96 & $96^{\circ}$ & 74 & 53 & 59 & 66 & 61 & 52 & 89 & 96 & 29 & 40 & 39 & 41 \\
\hline .01 & 58 & 77 & 92 & 95 & 83 & 51 & 43 & 55 & 17 & 21 & 31 & 94 & 18 & 19 & 26 & 33 \\
\hline \multicolumn{17}{|l|}{$\mathbb{E}, \mathbb{E}$} \\
\hline .05 & 83 & 100 & 120 & 121 & 55 & 77 & 107 & 106 & 51 & 36 & 50 & 92 & 29 & 50 & 56 & 59 \\
\hline .01 & 54 & 75 & $\mathbb{1 1 6}$ & 122 & 29 & 42 & 85 & 105 & 80 & 39 & 27 & 50 & 11 & 17 & 38 & 46 \\
\hline \multicolumn{17}{|l|}{$\mathrm{L}, \mathrm{L}$} \\
\hline .05 & 76 & 103 & 131 & 141 & 39 & 72 & 125 & 148 & 51 & 45 & 60 & 73 & 25 & 49 & 61 & 71 \\
\hline .01 & 34 & 67 & 122 & 142 & 13 & 29 & 85 & 132 & 70 & 50 & 43 & 45 & 6 & 15 & 42 & 59 \\
\hline \multicolumn{17}{|l|}{$D, \mathbb{D}$} \\
\hline .05 & 92 & 93 & 105 & 105 & 64 & 81 & 103 & 108 & 72 & 51 & 48 & 54 & 33 & 55 & 62 & 66 \\
\hline .01 & 60 & 89 & 103 & 110 & 39 & 55 & 95 & 109 & 108 & 49 & 39 & 41 & 10 & 21 & 53 & 59 \\
\hline \multicolumn{17}{|l|}{$\mathrm{C}, \mathrm{C}$} \\
\hline .05 & 67 & 94 & 131 & 139 & 20 & 38 & 78 & 102 & 52 & 82 & 99 & 108 & 21 & 50 & 76 & 91 \\
\hline .01 & 30 & 58 & 111 & 133 & 3 & 8 & 32 & 57 & 85 & 83 & 96 & 106 & 4 & 12 & 49 & 77 \\
\hline \multicolumn{17}{|l|}{$\mathrm{H}, \mathrm{H}$} \\
\hline .05 & 80 & 92 & 101 & 103 & 62 & 71 & 78 & 85 & 43 & 38 & 60 & 88 & 27 & 50 & 47 & 51 \\
\hline .01 & 57 & 80 & 96 & 103 & 49 & 54 & 65 & 79 & 54 & 33 & 30 & 65 & 11 & 18 & 35 & 41 \\
\hline \multicolumn{17}{|l|}{$M, M$} \\
\hline .05 & 82 & 91 & 104 & 105 & 57 & 66 & 88 & 97 & 68 & 62 & 65 & 71 & 50 & 50 & 52 & 58 \\
\hline .01 & 49 & 72 & 95 & 110 & 34 & 41 & 62 & 82 & 110 & 83 & 60 & 71 & 11 & 18 & 34 & 48 \\
\hline \multicolumn{17}{|l|}{$\mathbb{E}^{+}, \mathbb{E}^{-}$} \\
\hline .05 & 118 & 124 & 123 & 124 & 68 & 70 & 80 & 89 & 169 & 147 & 122 & 105 & 35 & 56 & 51 & 51 \\
\hline .01 & 135 & 173 & 176 & 155 & 73 & 61 & 73 & 83 & 170 & 196 & 127 & 92 & 31 & 34 & 48 & 44 \\
\hline
\end{tabular}

values to form a relative real power ratio. These ratios were converted to the nearest whole percent in Table 2, where a value greater than 100 indicates that an alternative PMC showed greater real power than $r_{\mathrm{P}}$ under the given conditions. In general, the agreement between the empirical normal distribution $(G, G)$ and the theoretical values $(T)$ is quite good except for $\gamma_{\mathrm{fp}}$ with $N<50$, where discontinuities distort the frequencies in the tails of its sampling distribution.

Although $r_{\mathrm{pb}}$ showed good relative real power $(>120 \%)$ for some distributional shapes when $Y$ was dichotomized at the sample median $\left(r_{\mathrm{pb} .5}\right)$, the loss in power was substantial under other conditions, especially with small sample sizes. By contrast, $r_{f p}$ showed poor relative real power for all population shapes under all conditions simulated.

Perhaps the most remarkable finding was the strong relative power performance of $r_{S}$ under a wide range of conditions. The advantage of the Spearman $r_{S}$ over the Pearson $r_{\mathrm{P}}$ appears to be greatest for markedly leptokurtic distributions such as the Cauchy and lognormal distributions. With $N=10$ the Pearson $r_{\mathrm{P}}$ tends to retain its power superiority regardless of kurtosis, but with $N=50$ and $N=100, r_{\mathrm{S}}$ is relatively more powerful than $r_{\mathrm{P}}$ for all of the distributions with excess kurtosis $(>3)$. In fact, the correlation is $r_{\mathrm{s}}=.90$ between the empirical distributions ranked by kurtosis, listed in Table 1, and the rank order of real relative power of $r_{s}$ at $N=50, \alpha=.05$ as given in Table 2. Thus, a choice of PMC statistic might very well depend upon knowledge of the peakedness of the parent $X, Y$ population. These 
Table 3

Enpirical and Theoretical Proportions of Rejections for Nomal Distribution $\rho=.1, N=20, \alpha \equiv .05$ Two-tailed

\begin{tabular}{|c|c|c|c|c|c|c|}
\hline \multirow[b]{2}{*}{ Statistic } & \multicolumn{4}{|c|}{ Empirical } & \multicolumn{2}{|c|}{ Theoretical } \\
\hline & Low & Median & High & S.D. & Mean & $\sigma$ \\
\hline \multicolumn{7}{|l|}{$\mathrm{sp}_{\mathrm{p}}$} \\
\hline Real power & .057 & .062 & .065 & .003 & .061 & .003 \\
\hline Type III & .007 & .008 & .010 & .001 & .008 & .001 \\
\hline \multicolumn{7}{|l|}{$r_{S}$} \\
\hline Rea1 power & .055 & .056 & .060 & .002 & .057 & .003 \\
\hline Type III & .008 & .009 & .011 & .001 & .008 & .001 \\
\hline \multicolumn{7}{|l|}{$I_{p b}$} \\
\hline Real power & .049 & .050 & .054 & .002 & .052 & .003 \\
\hline Type III & .011 & .012 & .012 & .001 & .010 & .001 \\
\hline \multicolumn{7}{|l|}{$r_{f p}$} \\
\hline Rea1 power & .038 & .040 & .043 & .002 & .023 & .002 \\
\hline Type III & .010 & .010 & .012 & .001 & .005 & .001 \\
\hline
\end{tabular}

results are also consistent with those of Wainer and Thissen (1976), who found a rank-order coefficient to be considerably more efficient than the Pearson coefficient in contaminated normal distributions.

When the individual distributions of two variables are not exactly the same shape (not necessarily normal), then the population correlation between them cannot reach the limits \pm 1 (Nunnally, 1978). When the population distributions of $X$ and $Y$ are markedly skewed in opposite directions, the maximum possible correlation between them is approximately \pm .7 (Carroll, 1961). One way of interpreting $r_{s}$ computed when sampling from $X$ and $Y$ distributions of different shapes is as an estimate of what the correlation between $X$ and $Y$ would be if the distributions were of identical shapes. One of the effects of ranking is, of course, to reduce the size of the interval between extreme scores and to increase the size of the interval between scores near the mode of a distribution. Thus, with light-tailed distributions having a preponderance of scores near the mode, such as the lognormal distribution, $r_{s}$ tends to expand the middle range more than it contracts the extremes. The result is an overestimate of $p$ which increases with $N$ for low values of $p$.

To obtain an estimate of the reliability of the simulation and performance of the computer random number generator, five successive runs of 5,000 cases each were conducted for the $G, G$ distribution with $N=20$. Comparisons between theoretical and empirical measures of central tendency and dispersion for $p=.1$ and $\alpha=.05$ are given in Table 3 , where the agreement is seen to be excellent for $r_{\mathrm{p}}, r_{\mathrm{S}}$, and $r_{\mathrm{pb}}$ and reasonable for $r_{\mathrm{fp}}$ due to the discontinuities in the distribution produced by the discrete categories with small sample sizes. The absolute rate of Type $\mathbb{I I I}$ errors is a concern only when both $p<.3$ and $N<20$, and relative rates are not meaningful at low values of total power.

There are, of course, many possible tradeoffs between power and parameter estimation. For example, for some population distributions, $p_{\mathrm{S}}$ might lead to more rejections of $\mathrm{H}_{0}$ than $r_{\mathrm{p}}$ with some combinations of $N$ and $p$, but the expected value of $r_{\mathrm{p}}$ may be closer to $p$ and the mean square error may be much less for $r_{\mathrm{P}}$ than for $r_{\mathrm{S}}$. A researcher could then decide whether magnitude of effect estimation was more important than null hypothesis testing. The present study was concerned only with the issues of relative real power and relative Type II error rates of PMCs. 


\section{Conchusions and Recommendations}

For graduated data, where a choice among PMC statistics exists, there appears to be no justification for using the phi coefficient. The possible exception to this would be where tables for rapid computation are available and the major concern is to minimize Type IIl errors when drawing small samples from extremely non-normal distributions.

The point-biserial correlation coefficient appears to offer some advantages over the Pearson $r$ when drawing samples of moderate size $(N>50)$ from populations that are either highly leptokurtic or highly skewed, as long as the dichotomization is near the median. Under these conditions, $r_{\mathrm{pb}}$ might also be computationally somewhat simpler than the Spearman coefficient in that only one variable need be ordered before applying the $\mathbb{P M C}$ formula. The major disadvantages of $r_{\mathrm{pb}}$ are the severe loss of real rellative power and the increase in Type III power in small samples which are drawn from most population distributional shapes.

In sampling graduated data from distributions other than bivariate normal, the Spearman rank-order correlation coefficient appears to be a viable alternative to the Pearson $r$ in terms of power. In fact, $r_{s}$ is not only robust but has the same or a greater degree of power than $r_{\mathrm{P}}$ with samples of moderate size $(N>50)$ from all distributions with excess kurtosis. However, as sample size increases, the computation of $r_{\mathrm{S}}$ becomes more laborious relative to $r_{\mathrm{p}}$, even for a computer, and the possibility of ties in the sample complicates the specification of a theoretical sampling distribution.

The Pearson product-moment correlation coefficient is remarkably robust and retains power under even extreme violations of distributional assumptions. Researchers should not be hesitant to report $r_{\mathrm{P}}$ under most circumstances as an index for $p$, although $r_{s}$ may sometimes be a more powerful test of the null hypothesis that $\rho=0$.

\section{References}

Box, G.E.P., \& Muller, M. (1958). A note on the generation of random normal deviates. Annals of Mathematical Statistics, 29, 610-611.

Bradley, J. V. (1968). Distribution-free statistical tests. Englewood Cliffs INJ: Prentice-Hall.

Bradley, J. V. (1977). A common situation conducive to bizarre distribution shapes. The American Statistician, 31, 147-150.

Carroll, I. B. (1961). The nature of the data, or how to choose a conrelation coefficient. Psychometrika, 26 , $347-372$.

Cohen, J. (1977). Statistical power analysis for the behavioral sciences (rev. ed.). New York: Academic Press.

Cohen, J. (1983). The cost of dichotomization. Applied Psychological Measurement, 7, 249-253.

David, $\mathrm{F} . \mathrm{N} .(1954)$. Tables of the ordinates and probability integral of the distribution of the correlation coefficien in small samples. Cambridge, England: Cambridge University Press.

Drasgow, $\mathbb{F}$., \& Dorans, N. I. (1982). Robustness of estimators of the squared multiple correlation and squared cross-validity coefficient to violations of mulivariate nomality. Applied Psychological Measurement, 6, 185-200.
Duncan, G. T., \& Layard, M. W. I. (1973). A montecarlo study of asymptotically robust tests for correlation coefficients. Biometrika, 60, 551-588.

Edgell, S. E., \& Noon, S. M. (1984). Effect of violation of normality on the test of the correlation coefficient. Psychological Bulletin, 95, 576-583.

Ferguson, G. A. (1981). Statistical analysis in psychology and education (5thed.). New York: McGrawHill.

Gayen, A. K. (1951). The frequency distribution of the product-moment correlation coefficient in random samples of any size drawn from non-normal universes. Biometrika, 38, 219-247.

Havlicek, L. L., \& Peterson, N. L. (1977). Effect of the violation of assumptions upon significance levels of the Pearson r. Psychological Bulletin, 84, 373377.

Hays, W. L. (1981). Statistics for the social sciences (3rd ed.). New York: Holt, Rinehart and Winston.

Kaiser, H. F. (1960). Directional statistical decisions. Psychological Review, 67, 160-167.

Kowalski, C. $\mathbb{J}$. (1972). On the effects of non-normality on the distribution of the sample product-moment correlation coefficient. Applied Statistics, Series $C, 21$, $1-12$. 
Kraemer, H. C. (1980). Robustness of the distribution theory of the product-moment correlation coefficient. Joumal of Educational Statistics, 5, 115-128.

Nunnally, J. C. (1978). Psychometric theory (2nd ed.). New York: McGraw-Hill.

Olsson, U., Drasgow, F., \& Dorans, N. J. (1982). The polyserial correlation coefficient. Psychometrika, 47, $337-347$.

Siegel, S. (1956). Nonparametric statistics. New York: McGraw-Hill.
Wainer, H., \& Thissen, D. (1976). Three steps towards robust regression. Psychometrika, 41, 9-34.

\section{A}

Send requests for reprints or further information to Robert $\mathbb{L}$. Fowler, Department of Psychology, University of South Florida, 140 Seventh Avemue South, Si. Petersburg FL 33701, U.S.A.

Downloaded from the Digital Conservancy at the University of Minnesota, http://purl.umn.edu/93227. 